\title{
Hobnail variant of papillary thyroid carcinoma showing goiter-like presentation and rapid growth
}

\author{
Anda Mihaela Naciu', Martina Verri², Anna Crescenzi ${ }^{2}$, Chiara Taffon², Filippo Longo3, \\ Luca Frasca3, Gaia Tabacco', Lavinia Monte1, Andrea Palermo'1, Pierfilippo Crucitti ${ }^{3}$ and \\ Roberto Cesareo 4
}

${ }^{1}$ Unit of Endocrinology and Diabetes, 2Unit of Pathology, ${ }^{3}$ Unit of Neck and Chest Surgery, Campus Bio-Medico Univerity of Rome, Rome, Italy, and 4Unit of Metabolic Diseases, 'S.M. Goretti' Hospital, Latina, Italy
Correspondence should be addressed to A Palermo

Email

a.palermo@unicampus.it

\section{Summary}

We present the case of a 47-year-old Caucasian previously healthy woman with a voluminous thyroid nodule occupying almost the entire anterior neck region. The lesion had progressively increased in size during the previous 3 months and the patient presented intermittent symptoms of dysphagia and odynophagia with a slight change in voice. Fine needle aspiration showed papillary carcinoma. Based on imaging and cytological findings, the patient underwent total thyroidectomy. The surgical sample revealed a totally enlarged thyroid gland (weight: $208 \mathrm{~g}$ ) with the presence of a polylobulated lesion centrally located and involving the isthmus and both lobes. Hobnail features were present in more than $30 \%$ of the neoplastic cells in agreement with the criteria for this subtype. Psammoma bodies and focal necrosis were also present. The extra-thyroidal extension included strap muscles and peri-esophageal glands. Immunohistochemistry using VE1 antibody for detecting BRAF-V600E mutation resulted positive. The final diagnosis was papillary thyroid carcinoma (PTC) hobnail variant (HVPTC)-pT4a. The HVPTC is a rare entity and, in most cases, appears like a unifocal lesion with a maximum tumor size of $8 \mathrm{~cm}$ reported so far. To our knowledge, this represents the largest tumor ever described $(14 \mathrm{~cm})$, showing rapid growth and with multinodular goiter-like aspect.

\section{Learning points:}

- HVPTC is an aggressive variant of PTC, usually associated with radioactive iodine refractoriness, and a higher mortality rate compared to classic PTC. However, there is a marked individual variability in this association.

- HVPTC usually appears as small unifocal lesion but a multinodular goiter presentation may occur.

- The present case highlights that despite of the histology, our patient achieved a high ablation success rate after radioactive iodine therapy.

\section{Background}

Papillary thyroid carcinoma (PTC) is the most common histological type of differentiated thyroid malignancy. Although mainly of the PTC variants do not pose a diagnostic challenge to pathologists, the real importance of identifying aggressive PTC variants is for the multidisciplinary management that requires more aggressive surgery, adjuvant therapy and closer surveillance (1). Herein, we report a case of hobnail variant of PTC (HVPTC) with the elaboration of its clinicopathologic features, and prognosis. The particularity of this case is the presentation as goiterlike and rapid growth with an unexpected response to radioactive iodine therapy and good prognosis. 

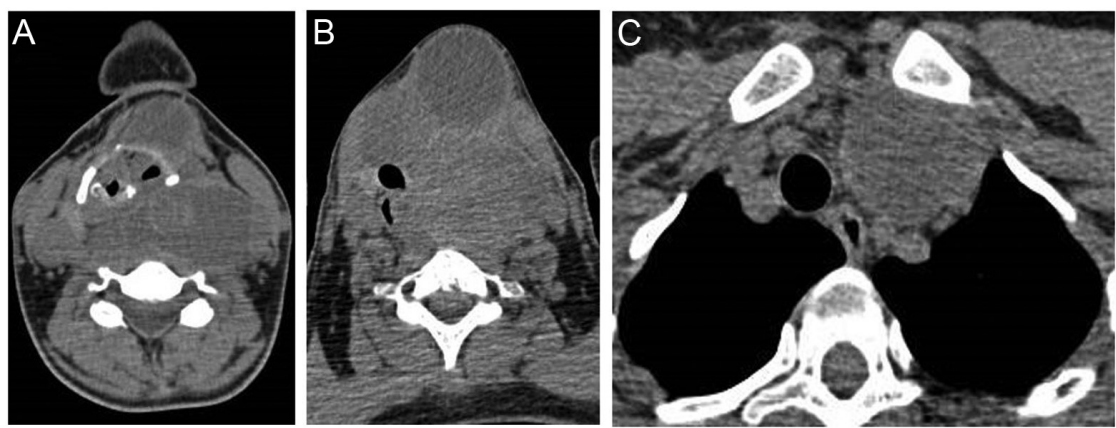

Figure 1

(A) Goiter compression of pharyngeal-laryngeal neck structures. (B) Tracheal-esophagus compression and lateral deviation. (C) Goiter extension to epiaortic vessels.

\section{Case presentation}

We present the case of a previously healthy 47-year-old Caucasian woman with a voluminous thyroid nodule occupying almost the entire anterior neck region. It was of acute onset and was noticed upon waking up from bed. It progressively increased in size during the previous 3 months. In the last 2 months she presented intermittent symptoms of dysphagia and odynophagia with a slight change in voice. However, there was no stridor or airway obstruction. She had no history of trauma, surgery to her neck, or insect bites. She was also not taking any traditional medications or anticoagulants. When she presented to our department, she was afebrile with normal vital signs.

Upon examination, there was a diffuse anterior neck swelling measuring around $15 \mathrm{~cm}$, which was firm and nontender. Blood tests showed normal levels of TSH, free T4, thyroid peroxidase antibody, thyroglobulin antibody serum and calcitonin.

\section{Investigation}

Neck ultrasound showed an enlarged thyroid gland with the presence of thyroid nodules with solid and cystic components and with retrosternal expansion.

Focusing on surgical planning, because of the reported rapid growth and on suspicion of an oncologic disorder, a neck-thorax CT scan was performed. CT scan showed the presence of a massive multinodular goiter extending along a plane passing through the soma of $\mathrm{C} 2$ down in the thorax to approximately $1.2 \mathrm{~cm}$ from the aortic arch, for a total extension of $14 \mathrm{~cm}$ and a transverse diameter of about $7.2 \mathrm{~cm}$. The mass exerted a compressive effect on trachea which was deviated to the right with an important lumen reduction. The same compressive effects were present in pharyngeal-laryngeal neck structures and on trachea (right deviation and important lumen reduction). No cervical lymphadenopathy was evident (Fig. 1A, B and C).

Fine needle aspiration showed cellular smears including fragments with papillary branching and thyrocytes, sometimes dischoesive, with clear irregular nuclei and intranuclear inclusion consistent with papillary carcinoma (PTC), TIR5 category (2) (Fig. 2).

\section{Treatment}

Based on imaging and cytological findings, the patient underwent total thyroidectomy. Once anestesiologist performed an oro-tracheal intubation with the use of a fibroscopic guide, as preoperatively planned, a wide Kocher cervicotomy was performed. Technically, there were no unexpected difficulties in approaching right thyroid lobe that presented multinodular but cleavvable from nearby structures. Left lobe mobilization was challenging: left recurrent nerve was strictly adherent to the gland and a pharingo-esophageal infiltration was evidenced in an attempt to dislocate the lobe; moreover, a meticulous and delicate laryngeal nerve isolation and preservation were

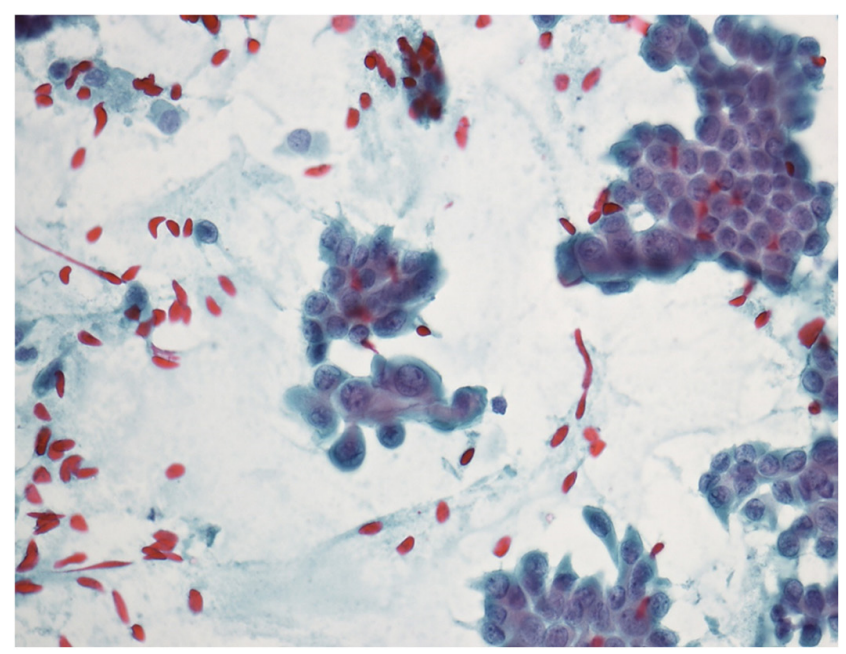

\section{Figure 2}

Fine needle aspiration consistent with papillary carcinoma. Crowded cells with nuclear overlapping, clear finely granular chromatin, irregular nuclear membranes, longitudinal grooves, and intranuclear inclusions. Discohesive cells with apically or eccentrically placed nuclei were easily recognized. 

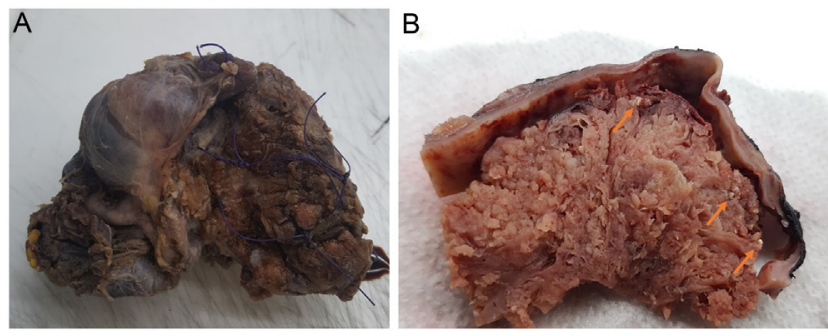

Figure 3

(A) Gross examination of formalin-fixed surgical sample. The gland was occupied by a large poly-lobulated lesion involving isthmus and both lobes with a max diameter of $12 \mathrm{~cm}$. The extra-thyroidal invasion into muscle and fat tissue was also evident. (B) The cut surface of the lesion showed a solid growth of neoplastic tissue with cystic changes. Solid areas appeared granular or papillary, brown, and microcalcifications were evident as single white dots or in cluster (arrows).

first performed. During thyroid isolation from pharingoesophageal junction, it was necessary to incise the pharingeal wall to achieve a radical dissection. Pharingoesophageal junction was then repaired manually.

The surgical sample revealed a totally enlarged thyroid gland (weight $208 \mathrm{~g}$ ) with the presence of a polylobulated lesion centrally located and involving the isthmus and both lobes (Fig. 3A). The cut surface showed an invasive tissue mass with cystic changes. Finger-like papillary neoplastic tissue contained microcalcifications (Fig. 3B). Histological examination showed a malignant neoplasm with papillary and micropapillary architecture. Hobnail features were present in more than 30\% of the neoplastic cells in agreement with criteria for this subtype (3) (Fig. 4A and B). Psammoma bodies and focal necrosis were also present. The extra-thyroidal extension included strap muscles and peri-esophageal glands. Immunohistochemistry using VE1 antibody for detecting BRAF-V600E mutation resulted positive (Fig. 5), in agreement with the presence of BRAF mutations in about $70-80 \%$ of these cases $(1,4)$. The final diagnosis was PTC hobnail variant (HVPTC)-pT4a (WHO $8^{\circ} \mathrm{Ed}(5)$ ).

\section{Outcome and follow-up}

Post-operative outcome: No intensive care was required. Patient immediately showed a left recurrent nerve deficiency confirmed by a laryngoscopy check. Initially the patient was kept fasting and refeeding was subordinated to a high gastrointestinal tract transit exam that was requested on post-operative day 5 . No complications were observed, and patient was discharged on post-operative day 8. Postoperatively, she had transient hypoparathyroidism that

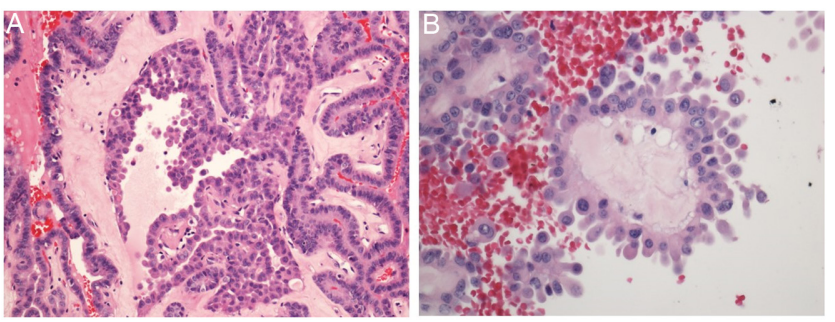

\section{Figure 4}

(A) Histological examination showed areas of classical type PTC (right side) associated with areas with micropapillary architecture in which neoplastic cells showed loss of cellular cohesion and eosinophilic cytoplasm (left side). Hematoxylin/eosin low power field. (B) Histology at high power field revealed typical hobnail features with fibro-vascular core covered with dischoesive neoplastic cells with apically located nuclei and prominent nucleoli. Hematoxylin/eosin low power field.

recovered within 3 weeks after treatment with calcitriol and supplemental calcium.

After surgery, patient received a therapeutic dose of $100 \mathrm{mCi}$ of radioactive iodine determining no tracer activity as seen on the pre-treatment diagnostic wholebody radioactive iodine scan. Actually, she is on levothyroxine replacement and after 3 years she has no evidence of disease.

\section{Discussion}

PTC is the most common histological type of differentiated thyroid malignancy (3) and it is usually associated with a good post-operative prognosis (4). However, among the numerous histological variants of PTC, the HVPTC

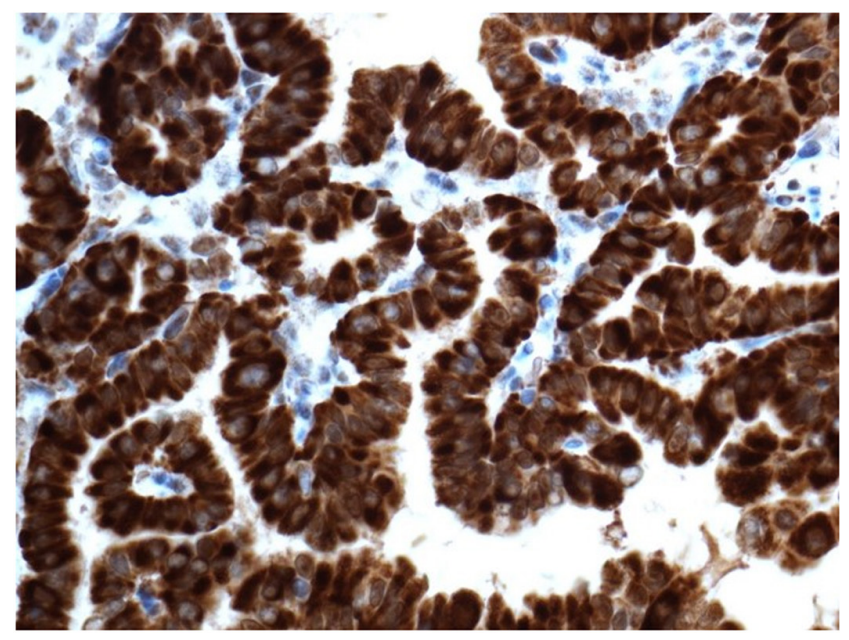

Figure 5

Immunohistochemistry using VE1 antibody detected BRAF V600E mutated protein. Brown reaction product is evident in the cytoplasm. Hematoxylin counterstained, low power field. 
is an aggressive form associated with radioactive iodine refractoriness, disease progression, and a higher mortality rate compared to classic PTC. Nevertheless, the HVPTC is a rare entity and, in most cases, appears like a unifocal lesion with a mean tumor size of $24 \mathrm{~mm}$ and a maximum tumor size described of $8 \mathrm{~cm}(1,4)$.

Diagnostic criteria of HVPTC have been better defined in the last version of World Health Organization Classification of Tumours of Endocrine Organs (3). The HVPTC is defined by $>30 \%$ of cells with hobnail features. This cut-off is higher compared with the previous rate reported therefore considered more representative of the aggressive variant. This variant of PTC is strongly associated with an extra-thyroidal invasion, in particular, metastasis to regional lymph nodes. However, our patient presented extra-thyroidal extension that included strap muscles and peri-esophageal glands.

In order to stratify the malignancy risk of thyroid nodule, thyroid imaging reporting and data systems (TIRADS) are used by ultrasound examination. TIRADS seems to be an accurate tool to diagnose PTC $(6,7,8)$ but has lower reliability in detecting non-typical PTC (7).

Previous studies identified BRAF p.V600E mutation as the most common alteration in HVPTC, as confirmed also in our case (4).

Despite the histological aspect and infiltration, our patient achieved a high ablation success rate after radioactive iodine treatment and during follow-up in our patient recurrency and distant organ metastasis have not yet occurred. After 36 months of follow-up she has no biochemical or imaging evidence of disease.

The deepening of molecular studies might be relevant to distinguish predictive parameters of the biological development of these aggressive tumors.

To our knowledge, this represents the largest tumor ever described $(14 \mathrm{~cm})$, showing rapid growth and multinodular goiter-like aspect.

\section{Declaration of interest}

The authors declare that there is no conflict of interest that could be perceived as prejudicing the impartiality of the research reported.

\section{Funding}

This research did not receive any specific grant from any funding agency in the public, commercial or not-for-profit sector.

\section{Author contribution statement}

A $M N, M V, P C$, and $R C$ involved in patient management, images preparation, manuscript writing, and manuscript editing. $F \mathrm{~L}, \mathrm{~L} F, \mathrm{~L} M, A P$ and $\mathrm{G} T$ involved in patient management and manuscript writing. $A C$ and C T contributed to pathological analysis and manuscript writing. A M N, M $\mathrm{V}, \mathrm{PC}$ and R C contributed equally to this work.

\section{References}

1 Watutantrige-Fernando S, Vianello F, Barollo S, Bertazza L, Galuppini F, Cavedon E, Censi S, Benna C, Ide EC, Parisi A, et al. The hobnail variant of papillary thyroid carcinoma: clinical/molecular characteristics of a large monocentric series and comparison with conventional histotypes. Thyroid 201828 96-103. (https://doi. org/10.1089/thy.2017.0248)

2 Nardi F, Basolo F, Crescenzi A, Fadda G, Frasoldati A, Orlandi F, Palombini L, Papini E, Zini M, Pontecorvi A, et al. Italian consensus for the classification and reporting of thyroid cytology. Journal of Endocrinological Investigation 201437 593-599. (https://doi. org/10.1007/s40618-014-0062-0)

3 Lloyd RV, Osamura RY, Klöppel G \& Rosai J. World Health Organization Classification of Tumours of Endocrine Organs, 4th ed., p. 355. Eds RV Lloyd, RY Osamura, G Klöppel \& J Rosai. International Agency for Research on Cancer, 2017.

4 Ambrosi F, Righi A, Ricci C, Erickson LA, Lloyd RV \& Asioli S. Hobnail variant of papillary thyroid carcinoma: a literature review. Endocrine Pathology 201728 293-301. (https://doi.org/10.1007/ s12022-017-9502-7)

5 Brierley JD, Gospodarowicz MK \& Wittekind C. TNM Classification of malignant tumours 8th edition. Wiley Blackwell and Union for International Cancer Control, 2016.

6 Lauria Pantano A, Maddaloni E, Briganti SI, Beretta Anguissola G, Perrella E, Taffon C, Palermo A, Pozzilli P, Manfrini S \& Crescenzi A. Differences between ATA, AACE/ACE/AME and ACR TI-RADS ultrasound classifications performance in identifying cytological high-risk thyroid nodules. European Journal of Endocrinology $2018 \mathbf{1 7 8}$ 595-603. (https://doi.org/10.1530/EJE-18-0083)

7 Trimboli P, Castellana M, Piccardo A, Romanelli F, Grani G, Giovanella L \& Durante C. The ultrasound risk stratification systems for thyroid nodule have been evaluated against papillary carcinoma. A meta-analysis. Reviews in Endocrine and Metabolic Disorders 2020. (https://doi.org/10.1007/s11154-020-09592-3)

8 Castellana M, Grani G, Radzina M, Guerra V, Giovanella L, Deandrea M, Ngu R, Durante C \& Trimboli P. Performance of EU-TIRADS in malignancy risk stratification of thyroid nodules: a meta-analysis. European Journal of Endocrinology 2020183 255-264. (https://doi.org/10.1530/EJE-20-0204)

Received in final form 20 December 2020

Accepted 7 January 2021 